\title{
Observation of Intermittent Transition by Electrode Biasing in Heliotron $\mathbf{J}^{*}$
}

\author{
Kosuke SHIMIZU, Sumio KITAJIMA, Atsushi OKAMOTO, Yu SATO, Jo TACHIBANA, \\ Toshihiro OKU, Masakazu TAKAYAMA ${ }^{1)}$, Fumimichi SANO ${ }^{2)}$, Tohru MIZUUCHI ${ }^{2}$, \\ Kazunobu NAGASAKI ${ }^{2}$, Hiroyuki OKADA ${ }^{2)}$, Shinichiro KADO ${ }^{2)}$, Shinji KOBAYASHI ${ }^{2}$, \\ Satoshi YAMAMOTO ${ }^{2)}$, Shinsuke OHSHIMA ${ }^{2)}$, Yasuhiro SUZUKI ${ }^{3)}$, Masayuki YOKOYAMA ${ }^{3)}$ \\ and Hiromi TAKAHASHI ${ }^{3)}$ \\ Department of Quantum Science and Energy Engineering, Tohoku University, Sendai 980-8579, Japan \\ 1) Akita Prefectural University, Honjyo, Akita 015-0055, Japan \\ ${ }^{2)}$ Institute of Advanced Energy, Kyoto University, Gokasho, Uji 611-0011, Japan \\ ${ }^{3)}$ National Institute for Fusion Science, 322-6 Oroshi-cho, Toki 509-5292, Japan
}

(Received 21 November 2014 / Accepted 3 March 2015)

\begin{abstract}
We observed intermittent increases in the electrode current in electrode biasing experiments in Heliotron J. In addition, electron density and floating potential showed pulsating behavior associated with the electrode current. The relation between line density and electrode current and that between floating potential and electrode current showed a hysteresis feature in transitions. Then it is evident that the pulsating behavior was the intermittent transition between two distinctive states. We also observed the mode $(\sim 10 \mathrm{kHz})$ that accompanied the pulsating behavior in the power spectrum density of the floating potential and ion saturation current obtained via fast Fourier transform. The electron density gradient increased, and subsequently the power spectrum density of the fluctuation increased.
\end{abstract}

(c) 2015 The Japan Society of Plasma Science and Nuclear Fusion Research

Keywords: stellarator, heliotron, electrode biasing, H-mode transition, turbulent transport, pulsation

DOI: $10.1585 /$ pfr. 10.3402061

\section{Introduction}

An electrode inserted in plasma generates radial current $\boldsymbol{J}$ and drives $\boldsymbol{J} \times \boldsymbol{B}$ poloidal flows, where $\boldsymbol{B}$ is the confinement magnetic field. Therefore, electrode biasing experiment is one of useful tools to inject an external torque into confined plasma and control bipolar transport to induce bifurcation of the radial electric field $E_{\mathrm{r}}$. In the Tohoku University Heliac, CHS, and LHD, the effect of viscosity maxima on the $\mathrm{L}-\mathrm{H}$ transition has been experimentally investigated by electrode biasing [1,2]. It is important to perform biasing experiments in a confinement system with configuration variability of the magnetic Fourier components, e.g., magnetic configuration in helical systems. Therefore, we have been continuing the electrode biasing experiments in Heliotron $\mathrm{J}$ to study the dependence of ion viscosity on helical ripples and bumpiness.

It is also important to observe the relation between fluctuation and radial electric field in order to understand turbulent transport, which is related to the onset of the $\mathrm{H}$ mode transition. The dynamic response of turbulence is one of the key issues to clarify the fundamental process of turbulent transport in limit cycle oscillations [3-7].

author's e-mail: kosuke.shimizu@pp12.qse.tohoku.ac.jp

*) This article is based on the presentation at the 24th International Toki Conference (ITC24)
In this study, we report the dynamic behavior of the transition induced by electrode biasing in Heliotron J. We observed the pulsating behavior accompanied with the low frequency fluctuation in low $\beta$ plasma. This allowed us to discuss the relation between the power spectrum of the fluctuation and radial electric field.

\section{Experimental Setup}

Heliotron $\mathrm{J}$ is a helical axis heliotron device with major radius $R_{0}=1.2 \mathrm{~m}$, minor radius $a=0.17 \mathrm{~m}$, and toroidal magnetic field $B_{0}<1.5 \mathrm{~T}$. The confinement magnetic field is produced by five sets of coils. The top view of Heliotron $\mathrm{J}$ is shown in Fig. 1. The biasing experiments were performed using a hot cathode electrode. The cylindrical hot cathode with diameter $10 \mathrm{~mm}$ and length $17 \mathrm{~mm}$ was made of $\mathrm{LaB}_{6}$ and located at $\rho=0.3$. The biasing system of Heliotron $\mathbf{J}$ is shown in Fig. 2. The target plasma for biasing was produced by the ECH $(f=2.45 \mathrm{GHz}$, $\left.P_{\max } \sim 19 \mathrm{~kW}\right)$ and the working gas was $\mathrm{H}_{2}(p=1.9 \times$ $10^{-6} \mathrm{~Pa}$ ) in the discharge-cleaning configuration (DCC) in Heliotron J. The strength of the magnetic field $B_{0}$, typical electron density $n_{\mathrm{e}}$, and electron temperature $T_{\mathrm{e}}$ on the magnetic axis were $\sim 0.09 \mathrm{~T}, \sim 2 \times 10^{17} \mathrm{~m}^{-3}$, and $\sim 30 \mathrm{eV}$, respectively. The electron density $n_{\mathrm{e}}$, temperature $T_{\mathrm{e}}$, and plasma potential were measured by the probe array shown 


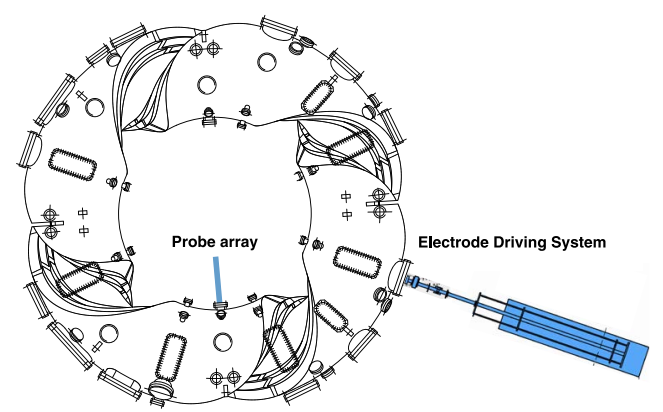

Fig. 1 Top view of Heliotron J. The hot cathode was inserted at $\phi=251.05^{\circ}$. Plasma parameters are measured with the probe array at $\phi=183.55^{\circ}$.

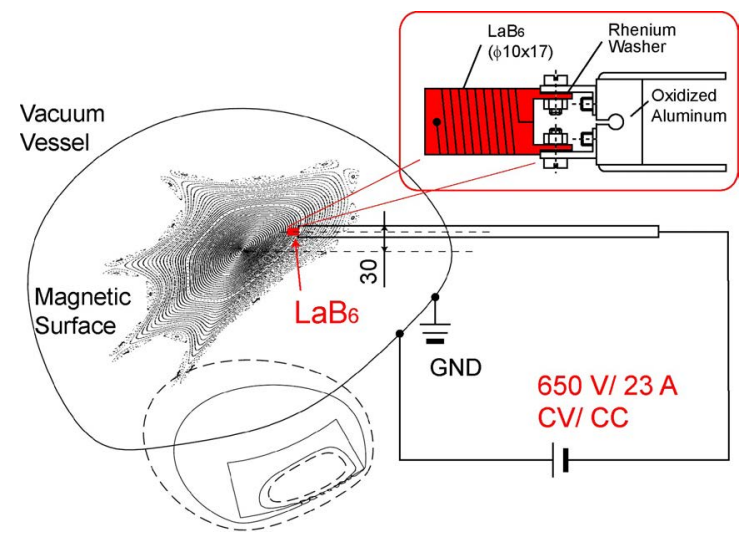

Fig. 2 The biasing system in Heliotron J. The hot cathode was made of $\mathrm{LaB}_{6}$ and located at $\rho=0.3$. The electrode was negatively biased against the vacuum vessel.

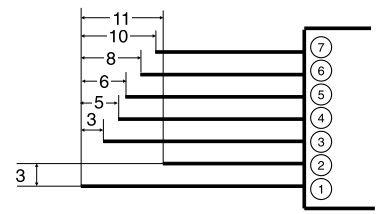

Fig. 3 Outline of the probe array.

in Fig. 3, which was designed to measure the floating potential at five different radial positions by probes $1,2,3,5$, and 7 [8]. Probes 1, 3, and 4 were used as a triple probe and probe 4 measured the ion saturation current.

\section{Experimental Results}

\subsection{Intermittent transition}

In the biasing experiments in Heliotron $\mathrm{J}$ using the electrode, the waveform for the bias voltage in the electrode current can be selected. Figure 4 shows the time evolutions of the electrode voltage (bias voltage) $V_{\mathrm{E}}$ and electrode current $I_{\mathrm{E}}$ through the electrode. The ramp-up and triangle waveforms were used in Figs. 4 (a) and (c). We observed intermittent increases in the electrode current in the voltage ramp-up stage in Fig. 4 (a) and ramp-down
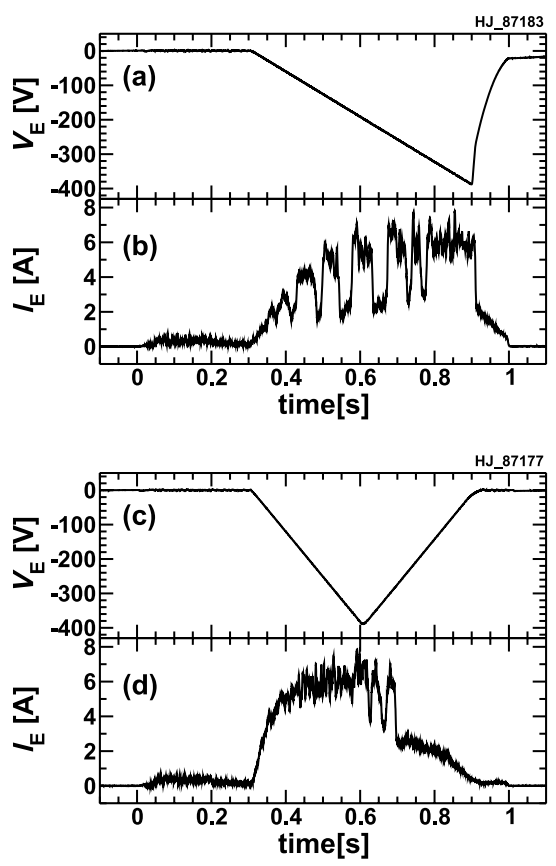

Fig. 4 Time evolutions of (a) and (c) electrode voltage (bias voltage) $V_{\mathrm{E}}$ and (b) and (d) electrode current $I_{\mathrm{E}}$.

stage in Fig. 4 (c). Then, to precisely observe the intermittent transition, we cautiously chose the operation conditions and successfully observed the same periodic features in the fixed voltage biasing $\left(V_{\mathrm{E}} \sim-100 \mathrm{~V}\right)$ shown in Fig. 5 . The biasing voltage was lower than that in Fig. 4. Figure 5 shows the time evolutions of the electrode voltage $V_{\mathrm{E}}$, electrode current $I_{\mathrm{E}}$, electron density $n_{\mathrm{e}}$, electron temperature $T_{\mathrm{e}}$, floating potential $V_{\mathrm{f}}$, and space potential $V_{\mathrm{s}}$. Clearly, the electrode current, electron density, and floating potential show periodicity in fixed voltage biasing. The period is about $80 \mathrm{~ms}$, and the rise and fall times are about $10 \mathrm{~ms}$ each. The pulsating behavior is slower than the pulsation observed in CHS [9]. In TEXTOR, intermittent transitions in the electrode voltage $V_{\mathrm{E}}$ and electrode current $I_{\mathrm{E}}$ were observed, and it was reported that the electric field gradient $\nabla E_{\mathrm{r}}$ was leading changes in electron density gradient $\nabla n_{\mathrm{e}}[10]$.

We chose the electron density and floating potential of two states of the periodic figure in the electrode current and plotted the radial profiles before and after crashes. The radial profiles of averaged density $n_{\mathrm{e}}$ and floating potential $V_{\mathrm{f}}$ are shown in Figs. 6 (a) and (b). The profile for the low electrode current state is close to the profile in L-mode and the profile for the high electrode current state is close to the profile in the improved confinement mode which obtained in a high voltage biasing.

In the biasing experiments, hysteresis was also observed between the electrode current and plasma parameters during the forward transition to the improved confinement mode and backward transition to the L-mode [11]. Figures 7 shows (a) relation between the line density $n_{\mathrm{e}} l$ 


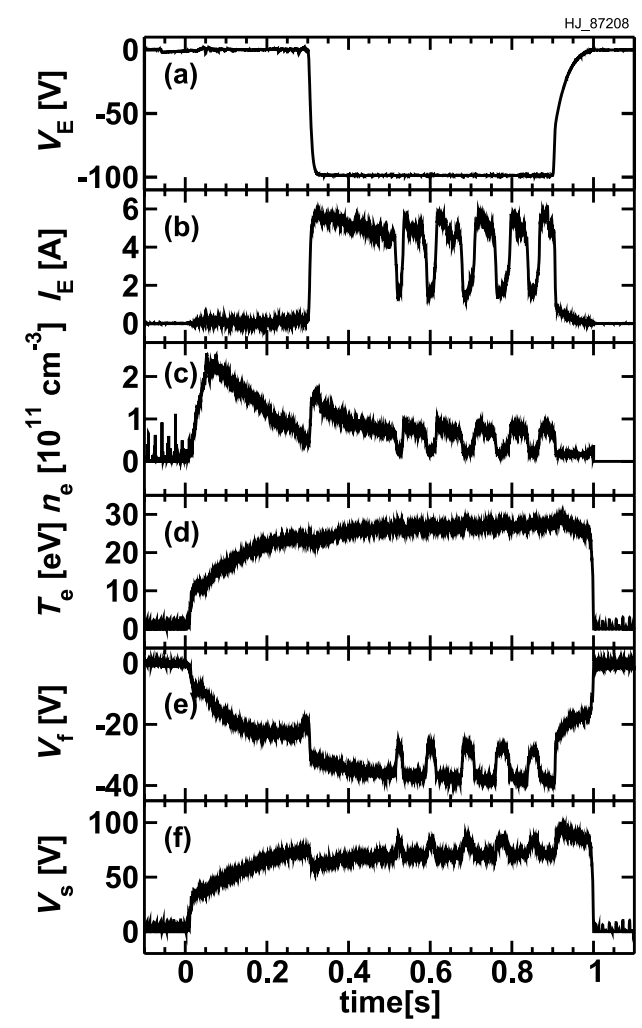

Fig. 5 Time evolutions of (a) the electrode voltage $V_{\mathrm{E}}$, (b) electrode current $I_{\mathrm{E}}$, (c) electron density $n_{\mathrm{e}}$, (d) electron temperature $T_{\mathrm{e}}$, (e) floating potential $V_{\mathrm{f}}$, and (f) space potential $V_{\mathrm{s}}$.

and electrode current $I_{\mathrm{E}}$, (b) relation between the floating potential $V_{\mathrm{f}}$ and $I_{\mathrm{E}}$, and (c) relation between the difference in electron densities $\Delta n_{\mathrm{e}}$ and $I_{\mathrm{E}}$ in the intermittent transition, respectively. The difference in electron densities $\Delta n_{\mathrm{e}}$ corresponds to the electron density gradient $\nabla n_{\mathrm{e}}$. The black and red lines show the transition to the high electrode current state and to low electrode current state, respectively. Figures 7 (a) and (b) clearly show the hysteresis and suggest that the periodic feature is the intermittent transition between the two distinctive states. In Fig. 7 (c), the difference of the electron densities was estimated from two averages of $n_{\mathrm{e}}$ data which had the same electrode current in the relation between $n_{\mathrm{e}}$ and $I_{\mathrm{E}}$. The $n_{\mathrm{e}}$ data were not measured simultaneously and were measured at two radial positions. The relation between $\Delta n_{\mathrm{e}}$ and $I_{\mathrm{E}}$ does not show hysteresis, suggesting that $\Delta n_{\mathrm{e}}$ is a single-valued function of $I_{\mathrm{E}}$.

\subsection{Low frequency mode in the high current state}

In the high electrode current state, a fluctuation of $\sim 10 \mathrm{kHz}$ was observed in the floating potential signal and the ion saturation signal measured by the triple probe. Figure 8 (a) shows the typical time evolution of the power spectrum density in the ion saturation current signal $I_{\mathrm{s}}$ obtained via fast Fourier transform. The figure clearly shows
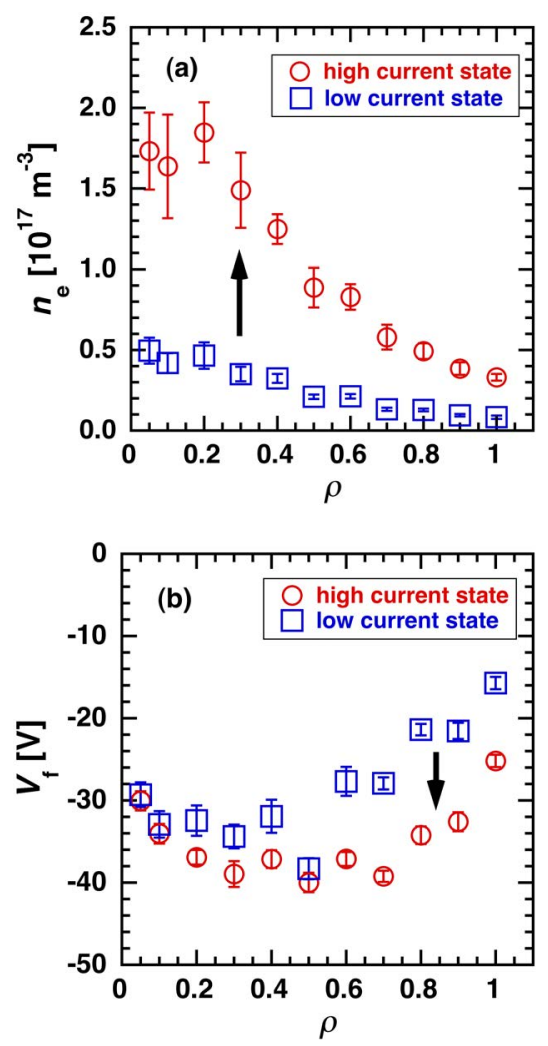

Fig. 6 Radial profiles of (a) averaged density $n_{\mathrm{e}}$ and (b) floating potential $V_{\mathrm{f}}$ before and after crashes.

that the power spectrum density increases in the wide frequency band in the high electrode current state compared with the low electrode current state. In particular, the new mode $(f \sim 10 \mathrm{kHz})$ occurs in the high electrode current state. Figure 8 (b) shows the time evolution of the power spectrum density in the floating potential $V_{\mathrm{f}}$. The new mode $(f \sim 10 \mathrm{kHz})$ is also observed in the high electrode current state, which may create channels for major heat and particle losses.

\subsection{Relation between power spectrum den- sity and electron density gradient}

Figure 9 shows the dependence of the power spectrum density of the fluctuation $(5<f<15 \mathrm{kHz})$ on the electron density difference between two radial positions $\Delta n_{\mathrm{e}}$ at $\rho=$ 0.5 and $\rho=0.6$. In Fig. 9, we plotted the power spectrum densities evaluated from five pulses in one discharge. After calculating the fitting curves for $I_{\mathrm{E}} \mathrm{vs} \Delta n_{\mathrm{e}}$, which represent the two transition curves between the two states in Fig. 7 (c), we adopted the electrode current $I_{\mathrm{E}}$ as the measure of the conditional average of $\Delta n_{\mathrm{e}}$ and power spectrum density. As seen in Fig. 9, the power spectrum density increased after the increase in $\Delta n_{\mathrm{e}}$, which corresponds to the electron density gradient. Lissajous-type dependence [7] can be observed between the power spectrum density and electron density gradient. 


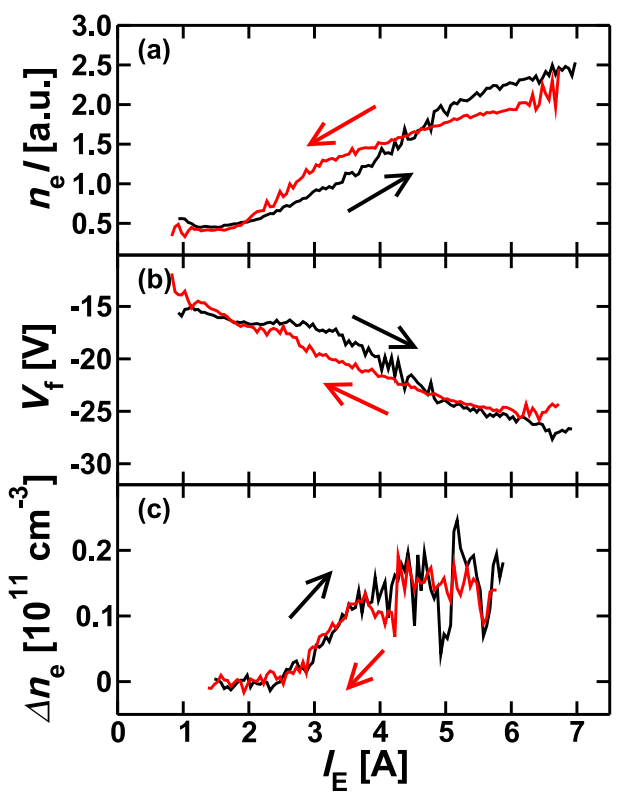

Fig. 7 (a) Relation between the line density $n_{\mathrm{e}} l$ and electrode current $I_{\mathrm{E}}$, (b) relation between the floating potential $V_{\mathrm{f}}$ and $I_{\mathrm{E}}$, and (c) relation between the difference in electron densities $\Delta n_{\mathrm{e}}$ and $I_{\mathrm{E}}$. The black and red lines denote the forward and backward transitions, respectively.

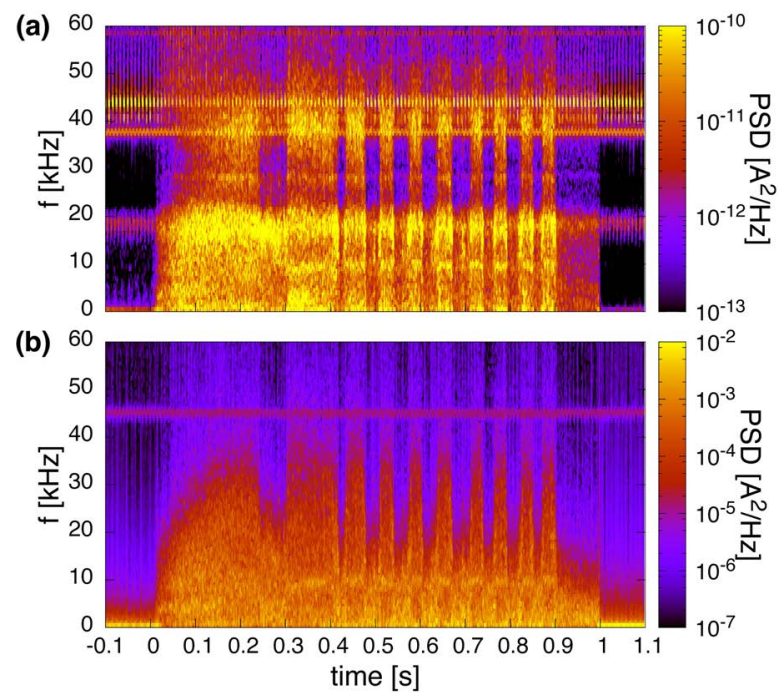

Fig. 8 Typical time evolution of (a) the power spectrum density of the ion saturation current signal $I_{\mathrm{s}}$ and (b) the floating potential $V_{\mathrm{f}}$ obtained via fast Fourier transform.

\section{Summary}

In the biasing experiments in Heliotron J, we observed intermittent increases in the electrode current. The density and floating potential also play a periodic feature according

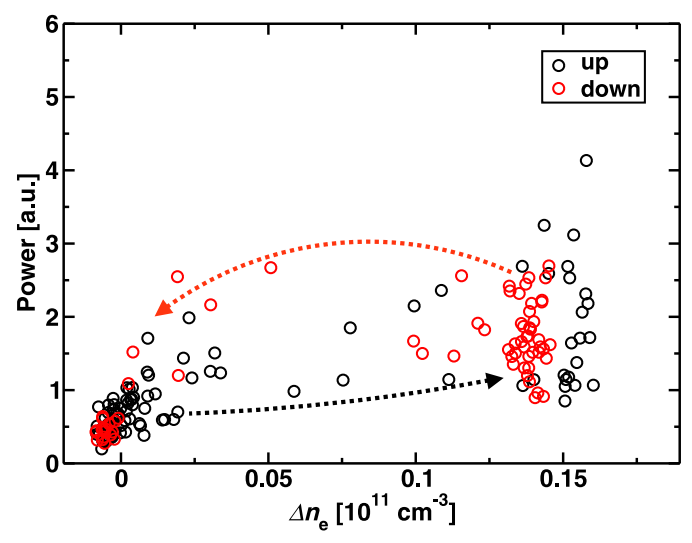

Fig. 9 Dependence of the power spectrum density of the fluctuation in the ion saturation current $I_{\mathrm{E}}$ on the electron density difference between the two radial positions $\Delta n_{\mathrm{e}}$.

to intermittent increases in the electrode current. The radial profiles of the averaged density and floating potential in two states before and after crashes are close to the profiles in L-mode and improved confinement mode, respectively. The relation between the line density and electrode current and that between the floating potential and electrode current suggest a hysteresis feature in transitions. The periodic features are the intermittent transition between two distinctive states. The power spectrum density of the fluctuation increased after the increase in electron density difference, which corresponds to the electron density gradient. We also observed the mode that accompanied the intermittent transition.

\section{Acknowledgment}

This work was performed with the support and under the auspices of the NIFS Collaboration Research Program (NIFS14KUHL063) and was partly supported by JSPS KAKENHI Grant Number 24246152.

[1] S. Kitajima et al., Nucl. Fusion 46, 200 (2006).

[2] S. Kitajima et al., Nucl. Fusion 53, 073014 (2013).

[3] K. Itoh et al., Plasma Fusion Res. 8, 1102168 (2013).

[4] G.D. Conway et al., Phys. Rev. Lett. 106, 65001 (2011)

[5] L. Schmitz et al., Phys. Rev. Lett. 108, 155002 (2012).

[6] J. Cheng et al., Phys. Rev. Lett. 110, 265002 (2013).

[7] T. Kobayashi et al., Phys. Rev. Lett. 111, 035002 (2013).

[8] S. Ohshima et al., Rev. Sci. Instrum. 81, 10E137 (2010).

[9] A. Fujisawa et al., Phys. Rev. Lett. 81, 2256 (1998).

[10] G. Van Oost et al., Plasma Phys. Control. Fusion 45, 621 (2003).

[11] H. Takahashi et al., Plasma Phys. Control. Fusion 48, 39 (2006). 